\title{
Video Article \\ On-chip Isotachophoresis for Separation of Ions and Purification of Nucleic Acids
}

\author{
Giancarlo Garcia-Schwarz ${ }^{1}$, Anita Rogacs ${ }^{1}$, Supreet S. Bahga ${ }^{1}$, Juan G. Santiago ${ }^{1}$ \\ ${ }^{1}$ Mechanical Engineering, Stanford University
}

Correspondence to: Juan G. Santiago at juan.santiago@stanford.edu

URL: https://www.jove.com/video/3890

DOI: doi:10.3791/3890

Keywords: Bioengineering, Issue 61, Isotachophoresis, electrokinetics, microfluidics, sample preparation

Date Published: 3/2/2012

Citation: Garcia-Schwarz, G., Rogacs, A., Bahga, S.S., Santiago, J.G. On-chip Isotachophoresis for Separation of lons and Purification of Nucleic Acids. J. Vis. Exp. (61), e3890, doi:10.3791/3890 (2012).

\section{Abstract}

Electrokinetic techniques are a staple of microscale applications because of their unique ability to perform a variety of fluidic and electrophoretic processes in simple, compact systems with no moving parts. Isotachophoresis (ITP) is a simple and very robust electrokinetic technique that can achieve million-fold preconcentration ${ }^{1,2}$ and efficient separation and extraction based on ionic mobility. ${ }^{3}$ For example, we have demonstrated the application of ITP to separation and sensitive detection of unlabeled ionic molecules (e.g. toxins, DNA, rRNA, miRNA) with little or no sample preparation $^{4-8}$ and to extraction and purification of nucleic acids from complex matrices including cell culture, urine, and blood. ${ }^{9-12}$

ITP achieves focusing and separation using an applied electric field and two buffers within a fluidic channel system. For anionic analytes, the leading electrolyte (LE) buffer is chosen such that its anions have higher effective electrophoretic mobility than the anions of the trailing electrolyte (TE) buffer (Effective mobility describes the observable drift velocity of an ion and takes into account the ionization state of the ion, as described in detail by Persat et al. ${ }^{13}$ ). After establishing an interface between the TE and LE, an electric field is applied such that LE ions move away from the region occupied by TE ions. Sample ions of intermediate effective mobility race ahead of TE ions but cannot overtake LE ions, and so they focus at the LE-TE interface (hereafter called the "ITP interface"). Further, the TE and LE form regions of respectively low and high conductivity, which establish a steep electric field gradient at the ITP interface. This field gradient preconcentrates sample species as they focus. Proper choice of TE and LE results in focusing and purification of target species from other non-focused species and, eventually, separation and segregation of sample species.

We here review the physical principles underlying ITP and discuss two standard modes of operation: "peak" and "plateau" modes. In peak mode, relatively dilute sample ions focus together within overlapping narrow peaks at the ITP interface. In plateau mode, more abundant sample ions reach a steady-state concentration and segregate into adjoining plateau-like zones ordered by their effective mobility. Peak and plateau modes arise out of the same underlying physics, but represent distinct regimes differentiated by the initial analyte concentration and/or the amount of time allotted for sample accumulation.

We first describe in detail a model peak mode experiment and then demonstrate a peak mode assay for the extraction of nucleic acids from $E$. coli cell culture. We conclude by presenting a plateau mode assay, where we use a non-focusing tracer (NFT) species to visualize the separation and perform quantitation of amino acids.

\section{Video Link}

The video component of this article can be found at https://www.jove.com/video/3890/

\section{Protocol}

\section{Physics of ITP}

ITP forms a sharp moving boundary between ions of like charge. The technique can be performed with anionic or cationic samples, but we tailor this introduction to anionic ITP and note the same principles apply to cationic ITP. We choose LE and TE buffers such that LE ions have higher magnitude effective electrophoretic mobility. The effective electrophoretic mobility, $\mu=U / E$, is the proportionality constant between applied electric field, $E$, and ion drift velocity, $U .{ }^{13}$ We establish a diffuse interface between the LE and TE and apply an electric field directed from the high-conductivity LE zone to the low-conductivity TE zone. The system quickly establishes a strong gradient in electric field at the ITP interface, due to the non-uniform conductivity profile. As per its name (from Greek, "isos" means "equal", "takhos" means "speed"), TE and LE ions travel at the same, uniform velocity, as a result of the non-uniform electric field and conservation of current (this is the so-called "ITP condition", see Figure 1).

The ITP interface is self-sharpening: LE ions that diffuse into the TE zone experience a strong restoring flux and return to the leading zone (and vice versa for TE ions in the LE zone). Sample ions focus at this interface if their effective mobility in the TE zone is greater than those of the TE co-ions, and if their effective mobility in the LE zone is less than that of the LE co-ions (see Figure 1). The self-sharpening and focusing 
properties of ITP contribute to the robustness of this technique and make ITP relatively insensitive to disturbances of the interface (e.g. due to pressure-driven flow or changes in geometry, such as contractions, expansions, and turns).

In peak mode ITP (see Figure 2a and Videos 1-2), sample ion concentrations are at all times significantly lower than LE and TE ion concentrations and therefore contribute negligibly to local conductivity. The distribution of sample ions is determined by the self-sharpening interface between neighboring zones (here the TE and LE) and the value of the sample effective mobility relative to these zones. ${ }^{14}$ Multiple sample ions focus within the same narrow ITP interface region as largely overlapping peaks. The interface and peak widths, as well as the associated preconcentration factor, scale inversely with the applied current (see experiments in Figure $\mathbf{2 b}$ ). ${ }^{14}$

For sufficiently high initial sample concentrations and sufficient accumulation time, sample ions reach a threshold concentration value. For fullyionized species, this value is determined by the Kohlrausch regulating function (KRF). ${ }^{15}$ For weak electrolytes, it is determined by the Alberty and Jovin functions. ${ }^{16,17}$ In plateau mode, as depicted in Figure $\mathbf{3}$ and Video 3, sample ions separate and purify into zones of locally uniform and constant concentration in an order determined by their effective mobility. Very dilute ions may still focus in peak mode between plateau zones. In ITP, sample ions can be introduced in a finite injection between the TE and LE (see Figure 3) or alternately mixed together with the TE and/ or LE (see Figure 2). We refer to mixing in the TE zone as "semi-infinite" injection, which can be used to accumulate analyte ions continuously. Continuous sample accumulation increases sensitivity in both peak and plateau mode assays. However, finite injections are more common in plateau mode. This is likely because high initial analyte concentrations in semi-infinite injection can substantially increase TE conductivity and lower focusing rates. Also, full purification is not possible in semi-infinite injection (since there remains a finite concentration in TE).

\section{Device cleaning and preparation}

For the assays presented in this protocol we use isotropically wet-etched (roughly D-shaped cross section) glass microfluidic chips with a crosschannel design (see Figure 1). The following cleaning and preparation protocol is optimized for borosilicate and fused silica channels, but can also be used with glass/PDMS chips. Perform this cleaning procedure prior to experiments to ensure run-to-run repeatability and successful application of dynamic coatings needed to suppress electroosmotic flow (EOF). Omission of this protocol may result in strong dispersion of the ITP interface. ${ }^{14}$

1. To decontaminate the channel, fill the North, East, and South reservoirs with $10-20 \mu \mathrm{L}$ of $10 \%$ bleach and apply vacuum at the West reservoir for $2 \mathrm{~min}$. If using a standard Caliper chip caddy, vacuum can be effectively applied by simply attaching the wide end of a $200 \mu \mathrm{L}$ (unfiltered) pipette tip to the chip reservoir and connecting the vacuum line to a $2 \mathrm{~mm}$ inner diameter tube.

2. Empty the reservoirs and rinse the channel (as in step 1) with $1 \mathrm{M}$ sodium hydroxide for $2 \mathrm{~min}$. This gently etches the channel walls, yielding a clean borosilicate surface to help establish uniform surface properties.

3. Empty the reservoirs and clean with de-ionized water (DI), then rinse the channel with LE for $\sim 2$ min. During this period surface properties and dynamic coatings equilibrate within the channel.

\section{Fluorophore focusing in peak mode ITP}

1. Prepare $1 \mathrm{~mL}$ LE consisting of $100 \mathrm{mM} \mathrm{HCl}, 200 \mathrm{mM}$ tris, and $1 \% \mathrm{PVP}$.

2. Prepare $1 \mathrm{~mL}$ TE consisting of $100 \mathrm{mM}$ HEPES and $200 \mathrm{mM}$ tris. Combine $90 \mu \mathrm{l}$ of TE with $10 \mu \mathrm{l}$ of $1 \mu \mathrm{M}$ Alexa Fluor 488 (AF488).

3. After rinsing with LE as described in Part 2, empty the West reservoir and clean a few times with DI in order to dilute any LE remaining in the reservoir. Fill this reservoir with $20 \mu \mathrm{L}$ TE containing AF488.

4. Place the positive electrode in the East reservoir and the ground (negative) electrode in the West reservoir and apply $2 \mu \mathrm{A}$ (constant current). The sample peak will migrate at a constant velocity from the West reservoir to the East reservoir (see Figure 1) and the voltage between these reservoirs will increase as the lower conductivity TE fills the channel.

\section{Extraction and purification of nucleic acids from cultured $E$. coli}

The ability to selectively focus ionic species makes ITP an ideal technique for biological sample preparation. We purify nucleic acids from untreated cell lysate by selecting a trailing anion with an effective mobility magnitude lower than the target nucleic acid but higher than co-ionic PCR inhibitors (e.g. anionic detergents, proteins, and organic solvents, even if present in high concentration). Cationic PCR inhibitors (e.g. alkali metals and cationic proteins and detergents) migrate in the opposite direction and so are also left behind. ITP extracts and focuses target nucleic acids from the sample reservoir, while leaving slower PCR-inhibiting species behind (see Figure 4).

1. Obtain a sample of or culture $E$. coli cells to a density greater than $10^{8} \mathrm{CFU} / \mathrm{mL}$.

2. Transfer $1 \mathrm{~mL}$ cell culture into a safe-lock microcentrifuge tube and pellet by centrifugation at $4000 \mathrm{~g}$ for $6 \mathrm{~min}$.

3. Re-suspend the pellet in $80 \mu \mathrm{L}$ RNase-free water and add $10 \mu \mathrm{L}$ of lysing agent consisting of $10 \mathrm{mM}$ tricine, $10 \mathrm{mM}$ bis-tris, $2 \mathrm{mM}$ EDTA, 0.1 $\%$ Triton-X, and $5 \mathrm{mg} / \mathrm{mL}$ lysozyme. Mix gently and incubate for 5 mins. at room temperature (lysing protocol adapted from Bercovici et al. ${ }^{11}$ ).

4. Add $10 \mu \mathrm{L}$ of $1 \mathrm{M}$ sodium hydroxide to raise the lysate $\mathrm{pH}$ to $\sim 12.5$. Gently actuate the pipette up and down until the solution becomes clear, at which point lysing is complete.

5. Combine $10 \mu \mathrm{L}$ of lysate with $90 \mu \mathrm{L}$ of $50 \mathrm{mM}$ tricine and $100 \mathrm{mM}$ bis-tris. This solution can now be used as the TE.

6. Prepare $1 \mathrm{~mL}$ of LE consisting of $500 \mathrm{mM}$ bis-tris, $250 \mathrm{mM} \mathrm{HCl}, 1 \% \mathrm{PVP}$, and $1 \mathrm{X}$ SYBR Green II. Fill the microfluidic chip with LE as described in Part 3.

7. In order to extract the purified nucleic acid for off-chip analysis following ITP, replace the contents of the East reservoir with a PCR-compatible LE containing $50 \mathrm{mM}$ bis-tris, $25 \mathrm{mM} \mathrm{HCl}$, and $0.1 \%$ PVP. Apply $1000 \mathrm{~V}$ between the East and West wells to begin the experiment. The current between these reservoirs will decrease.

8. At the end of the experiment the sample elutes into the LE reservoir. Coincident with this elution, the current versus time for this system typically reaches a plateau value (since resistance is now dominated by TE ions uniformly distributed within channel). Gently mix the reservoir contents by repeated pipetting and extract a $5 \mu \mathrm{L}$ volume for analysis by quantitative RT-PCR. 


\section{Separation of amino acids with cationic plateau mode ITP and non-focusing tracer (NFT) for visualization}

ITP can be used to separate and focus small ions into adjoining and detectable plateaus between the TE and LE. This allows detection and identification based on physicochemical properties, such as local conductivity, UV absorbance, temperature sensing, or index of refraction. Here we demonstrate a non-focusing tracer (NFT) assay where a fluorescent, co-ionic species is added to the LE. This fluorescent species does not focus, but its concentration adapts to a local electric field and thereby enables visualization of purified plateau zones (see Figure 5).

1. Prepare $1 \mathrm{~mL}$ LE consisting of $100 \mathrm{mM}$ ethanolamine, $200 \mathrm{mM}$ tricine, and $1 \% \mathrm{PVP}$, and $100 \mu \mathrm{M}$ of the cationic fluorophore Rhodamine $6 \mathrm{G}$.

2. Prepare $1 \mathrm{~mL}$ TE consisting of $20 \mathrm{mM}$ tris, $40 \mathrm{mM}$ tricine. Prepare sample by mixing $90 \mu \mathrm{L}$ of TE with $10 \mu \mathrm{L}$ each of $50 \mathrm{mM}$ arginine and 50 $\mathrm{mM}$ lysine.

3. Dispense $20 \mu \mathrm{L}$ LE in the West and North reservoirs and sample in the East reservoir. Apply vacuum at South reservoir for $1 \mathrm{~min}$.

4. Rinse the East well with DI and replace with TE (TE without sample).

5. Apply $500 \mathrm{~V}$ between the East and West reservoirs.

\section{Representative Results}

We show isotachopherograms of peak mode experiments in Figure $\mathbf{2 b}$ and nucleic acid extraction experiments in Figure $\mathbf{4}$. In peak mode experiments with a fluorescent reporter (e.g. AF488, SYBR Green II), the overall fluorescence intensity can be integrated and compared against a calibration curve to obtain quantitative concentration information. ${ }^{12}$ Additionally, in nucleic acid extraction experiments, sample is allowed to elute into the LE reservoir and extracted with a pipette for analysis by quantitative RT-PCR. ${ }^{10,12}$ We show plateau mode isotachopherograms for amino acid separation in Figure 5. Fluorescence intensity (relative to LE or TE zone intensity) can be used for zone identification, while zone widths enable quantitation.

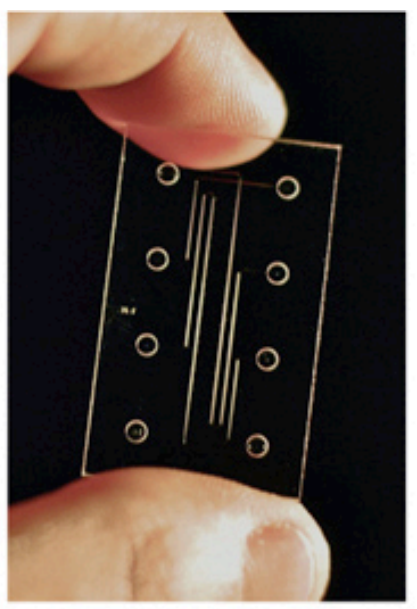

\section{Isotachophoresis (ITP):}

- Separation by ionic mobility

- Selective focusing

- 10,000-fold preconcentration

- Insensitive to disturbances
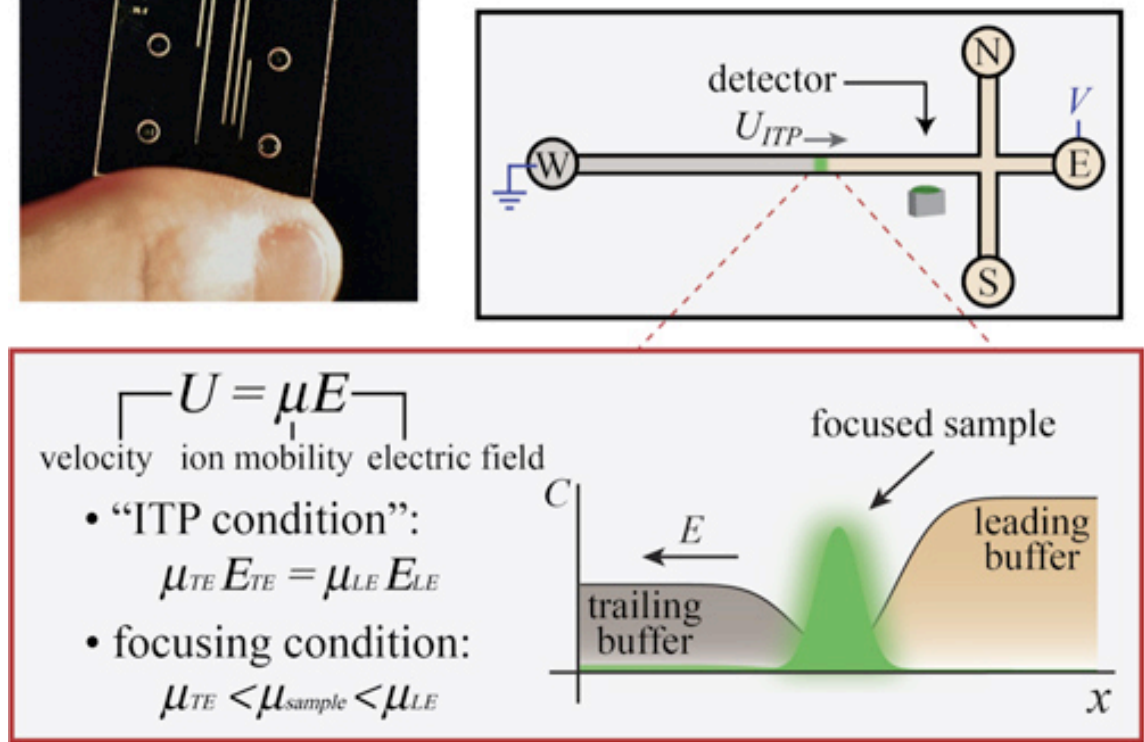

Figure 1. Isotachophoresis (ITP) is an electrokinetic technique used in microfluidic applications for sensitive detection and separation of ions. ITP offers separation, selective focusing, and preconcentration capabilities. In addition, it is extremely robust and insensitive to physical disturbances because of its self-sharpening nature. Sample ions selectively focus between a leading (LE) and trailing electrolyte (TE) and travel through the microchannel at a constant speed determined by the speed of the leading ions in the LE zone. Sample ions focus if their effective electrophoretic mobility is bracketed by the effective mobility of the LE and TE ions. The schematic depicts a model ITP experiment where sample focuses continuously between the LE and TE zones. 


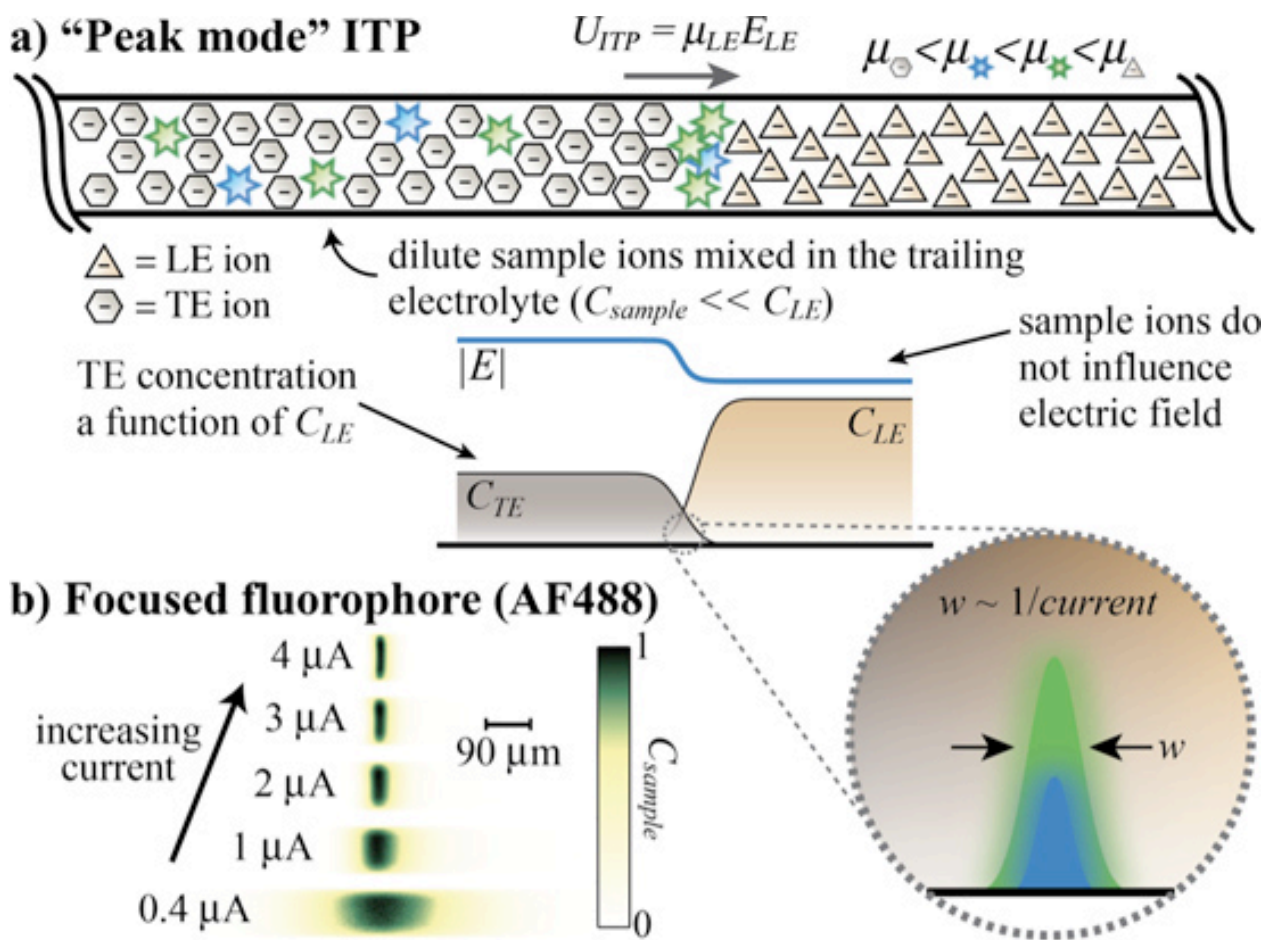

Figure 2. Dilute sample ions focus in "peak mode" ITP. a) Two distinct dilute ( $\left.c_{\text {sample }}<<c_{L E}\right)$ species focus in peak mode at the interface formed between the LE and TE zones. Solely the LE and TE determine the electric field, as sample species do not significantly contribute to current. Sample ions are mixed with TE (in a semi-infinite injection) and focus together in a roughly Gaussian peak at the ITP interface. Focusing criterion (shown as inequalities) applies to strongly ionized species. b) Experiments showing Alexa Fluor 488 (AF488) focused in peak mode for a range of applied currents (see also Video 1). Sample peak width is inversely proportional to current (for negligible advective dispersion due to electroosmotic flow). ${ }^{14}$ The self-sharpening interface is resistant to dispersion due to pressure-driven flow (see Video 2).

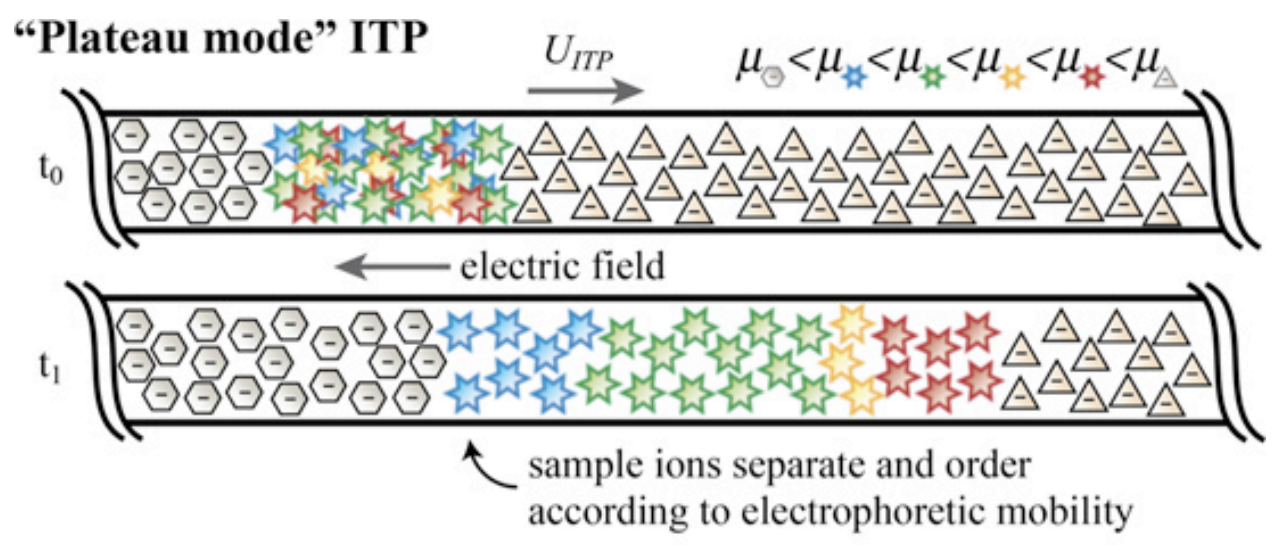

sample ions carry current

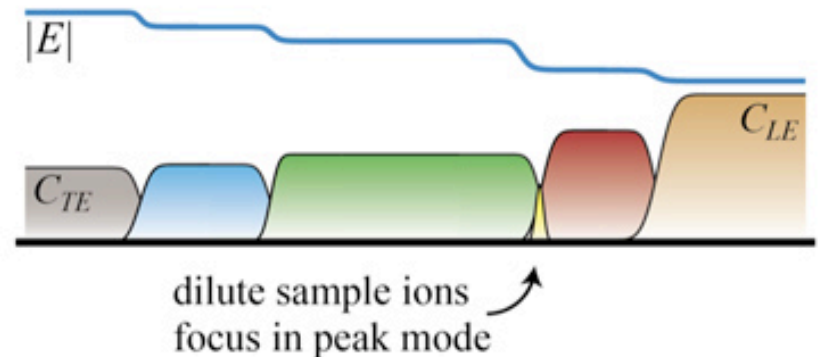

Figure 3. Sample ions at a sufficiently high concentration focus in "plateau mode" and govern local conductivity. We typically introduce sample ions in a finite injection between the TE and LE. In this modality, sample ions separate and order according to their effective electrophoretic mobility (see Video 3). For strongly ionized species, the TE and plateau zone concentrations are determined by the Kohlrausch regulating function (KRF, an invariant set initially by the LE zone). Dilute ions continue to focus in peak mode between plateau zones bracketing their effective mobility. Focusing criterion (shown as inequalities) applies to strongly ionized species. 

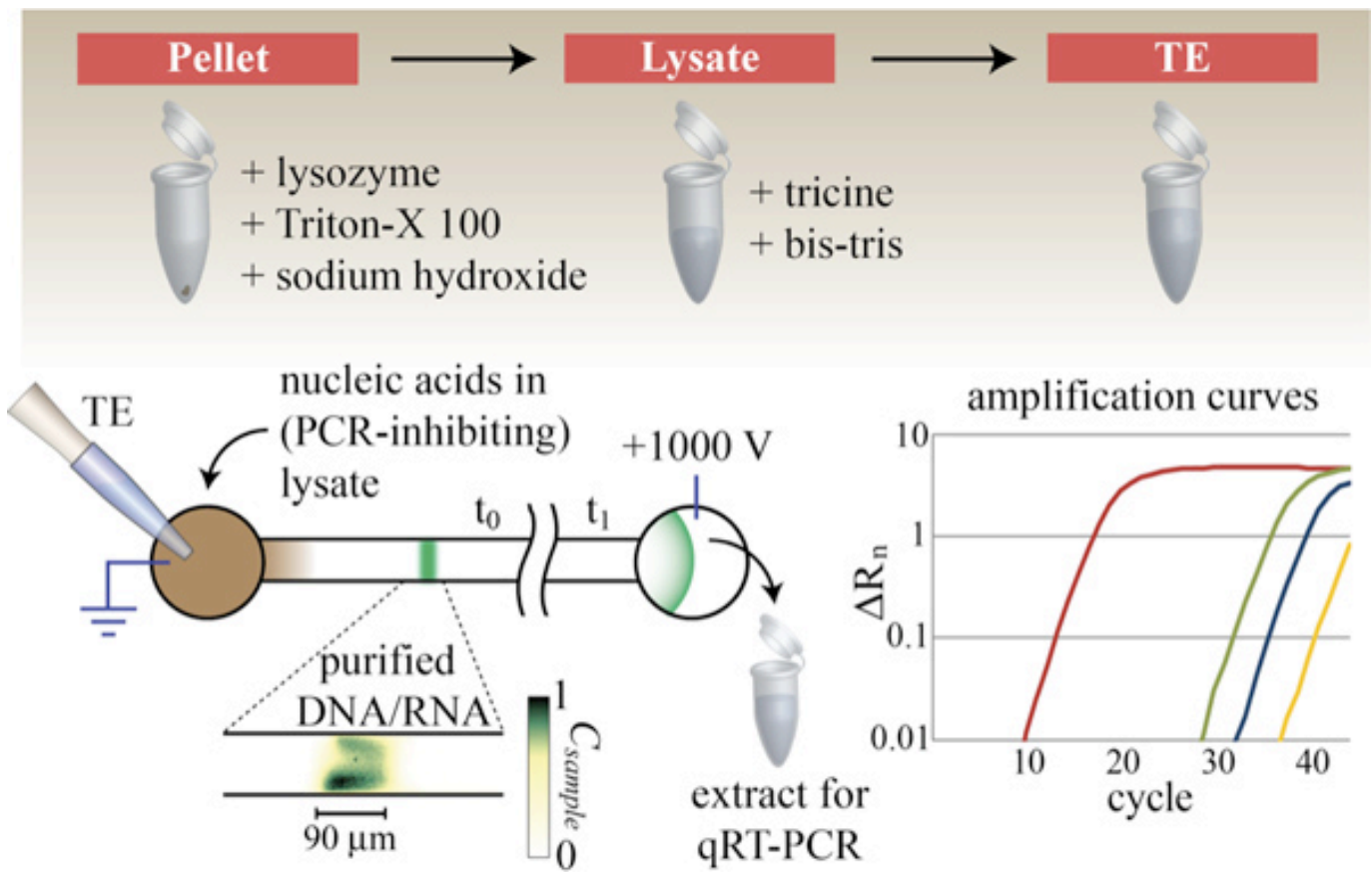

Figure 4. Lysate preparation and nucleic acid extraction with peak mode ITP. Nucleic acid is extracted from E. coli cell culture using lysozymeassisted alkaline lysing and purified by selective electrophoretic focusing via ITP. TE mixed with lysate (brown) contains target nucleic acid (green), proteins, and potential PCR-inhibiting chemistries. Appropriate selection of trailing and leading ions enables selective focusing of target nucleic acid while leaving PCR inhibitors behind. Total nucleic acid ITP peak often takes on a non-ideal shape, as shown here in the inset image. As a demonstration of this assay, we extracted total nucleic acid from gram-negative bacteria, Escherichia coli (lysed with sodium hydroxide solution alone), purified NA from lysate using ITP, collected the extracted genetic material, and performed qRT-PCR analyses to verify successful purification of 16S rRNA (red) and 16S rDNA (green) from bacterial culture. Negative control threshold cycles for 16S rRNA (blue) and 16S rDNA (yellow) were each above 30 cycles. We performed qRT-PCR using Power SYBR Green RNA-to-C $\mathrm{C}_{\mathrm{T}}$ 1-Step Kit from Applied Biosystems with $150 \mathrm{nM}$ forward (5'-CGGATTGGAGTCTGCAACTCG) and reverse (5'-CACAAAGTGGTAAG CGCCCTC) primers, at the recommended thermal cycling conditions. 


\section{a) Non-focusing tracer (NFT) assay}

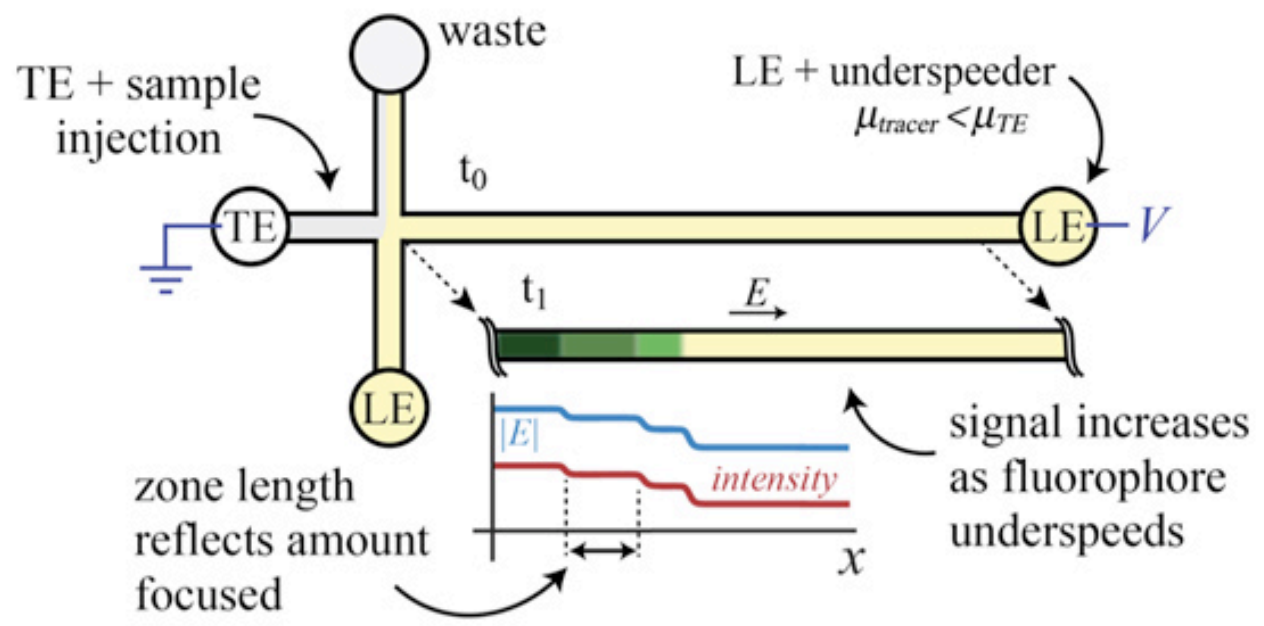

\section{b) Separation of amino acids with cationic ITP}

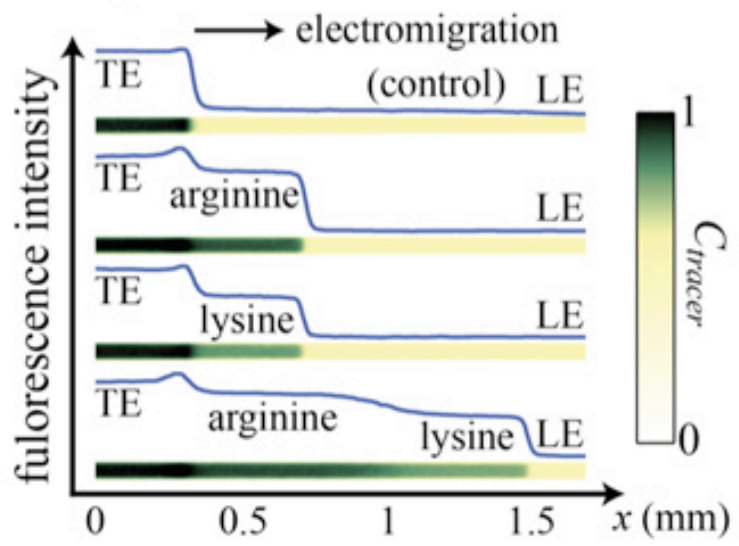

\section{LE: $100 \mathrm{mM}$ ethanolamine} $200 \mathrm{mM}$ tricine

TE: $20 \mathrm{mM}$ tris

$40 \mathrm{mM}$ tricine

Tracer: $200 \mu \mathrm{M}$ Rhodamine $6 \mathrm{G}$

Figure 5. Non-focusing tracer (NFT) assay for separation and detection of unlabeled amino acids. a) Schematic of the NFT assay. A finite injection of sample ions is introduced into the East channel. We mix the LE with a tracer cationic fluorophore with effective mobility lower than the TE ions $\left(\mu_{\text {tracer }}<\mu_{\mathrm{TE}}\right)$. The fluorophore is said to act as an "underspeeding tracer". As the fluorophore electromigrates from the LE into zones now occupied by sample plateaus and TE, it experiences a higher electric field and thus its concentration increases. This change in concentration creates steps in the isotachopherogram. b) Separation of two amino acids, arginine and lysine, using the NFT assay with Rhodamine $6 \mathrm{G}$ as the underspeeding fluorescent tracer. The slight peak between TE and arginine is common in ITP, is not well understood, and here does not interfere with detecting or quantifying the plateaus.

Supplemental Movie 1. Click here to view the supplemental movie.

Supplemental Movie 2. Click here to view the supplemental movie.

Supplemental Movie 3. Click here to view the supplemental movie.

\section{Discussion}

The ITP methods presented here enable rapid, sensitive, and robust detection and handling of ionic molecules. The main challenge in using ITP is proper choice of LE and TE buffers. In anionic ITP, we typically choose chloride as the leading anion because it is a strong acid with very high absolute mobility and thus has very predictable properties. Therefore, buffer choice in anionic ITP is typically reduced to choosing a proper TE and counterion. For selective focusing experiments, TE choice is critical to exclusion of contaminating ions. In applications where contaminants are not present, lower TE effective mobility leads to faster rate of focusing. We recommend using the following, relatively well-behaved anionic TE ions: MES, MOPS, HEPES, tricine. Choice of counterion affects both the system pH and TE effective mobility. Common counterions are tris (pKa 8.1) and bis-tris (pKa 6.4). For assays requiring lower or higher pH, we recommend pyridine (pKa 5.25) and ethanolamine (pKa 9.5), respectively. In Tables 1 and 2, for anionic and cationic ITP, respectively, we summarize several useful buffer examples. We take HCl as the anionic LE ion and sodium as the cationic LE ion, and we assume $100 \mathrm{mM}$ ionic strength of the LE buffer. 
The reader will note that buffer ionic strength in our protocols (and in Tables 1-2) is always greater than or equal to $10 \mathrm{mM}$. While in theory the physics of ITP applies at ionic strength lower than $10 \mathrm{mM}$, natural contaminants (e.g. carbonic acid from reaction between water and atmospheric carbon dioxide) near the $1 \mathrm{mM}$ level often limit the practical use of low ionic strength buffers.

We note that signal transduction mechanism is not limited to the assays presented in this protocol. As discussed briefly in Part 5 , in plateau mode purified zones can be detected via changes in local conductivity, UV absorbance, temperature, or index of refraction. In our own group, we have developed a method which utilizes fluorescent carrier ampholytes for sensitive detection and identification of unknown analytes. ${ }^{5}$ In peak mode experiments, specific probes can be used to label focused analytes. In one example, we use molecular beacons - oligonucleotide probes that fluoresce upon hybridization to their target sequence - to detect target DNA or RNA molecules with high specificity. ${ }^{11,18}$

While ITP is relatively robust to dispersion caused by pressure-driven flow and EOF, excessive EOF can lead to stagnation of the ITP interface, where the mean EOF velocity becomes equal to the ITP velocity. ${ }^{14}$ Such strong EOF occurs especially in conditions of high pH and low ionic strength. For this reason, we recommend the use of buffers with $\mathrm{pH} 8$ or lower and with ionic strength on the order of $100 \mathrm{mM}$ if convenient. In our experience, PVP is the most effective coating for EOF suppression in borosilicate chips. However, because of its sieving properties, PVP may not be appropriate for some applications, such as focusing genomic DNA. In experiments, we observe that addition of PVP can greatly reduce genomic DNA absolute mobility (to the point where it does not focus). In these cases a silanol coating such as Sigmacote in conjuction with a surfactant (e.g. Triton-X 100) can also be effective in reducing EOF. ${ }^{10}$

\begin{tabular}{|l|l|l|l|l|}
\hline & \multicolumn{5}{|l|}{ TE anion (pKa -1) } & HEPES (7.50) & tricine (8.15) \\
\hline $\begin{array}{l}\text { Buffering counterion (pKa } \\
\text { (1) }\end{array}$ & MES (6.10) & MOPS (7.20) & 17.38 & 22.85 \\
\hline ethanolamine (9.50) & 21.41 & 20.40 & 15.84 & 17.56 \\
\hline tris (8.08) & 21.00 & 19.23 & 7.30 & 5.23 \\
\hline bis-tris (6.40) & 18.22 & 10.41 & 2.42 & 1.48 \\
\hline pyridine (5.18) & 1.01 & 3.72 & \\
\hline
\end{tabular}

Table 1. Effective mobility magnitude $\left(\times 10^{-9} \mathrm{~m}^{2} / \mathrm{V} / \mathrm{s}\right)$ of adjusted pure analyte zone in anionic ITP where the LE is $100 \mathrm{mM} \mathrm{HCl}$ and $200 \mathrm{mM}$ buffering counterion.

\begin{tabular}{|l|l|l|l|l|}
\hline & \multicolumn{4}{|l|}{ TE cation (pKa +1) } \\
\hline $\begin{array}{l}\text { Buffering counterion } \\
\text { (pKa -1) }\end{array}$ & ethanolamine (9.50) & tris (8.08) & bis-tris (6.40) & pyridine (5.18) \\
\hline MES (6.10) & 35.57 & 21.96 & 16.39 & 10.45 \\
\hline MOPS (7.20) & 35.47 & 20.92 & 9.76 & 3.93 \\
\hline HEPES (7.50) & 35.35 & 19.97 & 7.77 & 2.88 \\
\hline Tricine (8.15) & 34.77 & 16.72 & 4.50 & 1.44 \\
\hline
\end{tabular}

Table 2. Effective mobility magnitude $\left(\times 10^{-9} \mathrm{~m}^{2} / \mathrm{V} / \mathrm{s}\right)$ of adjusted pure analyte zone in cationic ITP where the LE is $100 \mathrm{mM}$ sodium and $200 \mathrm{mM}$ buffering counterion.

\section{Disclosures}

We have nothing to disclose.

\section{Acknowledgements}

We gratefully acknowledge funding from DARPA sponsored Micro/Nano Fluidics Fundamentals Focus (MF3) Center under contract number N66001-10-1-4003, and from DARPA grant N660001-09-C-2082.

\section{References}

1. Jung, B., Bharadwaj, R., \& Santiago, J.G. On-chip millionfold sample stacking using transient isotachophoresis. Anal. Chem. 78 (7), 2319 (2006).

2. Jung, B., Zhu, Y., \& Santiago, J.G. Detection of 100 am fluorophores using a high-sensitivity on-chip CE system and transient isotachophoresis. Anal. Chem. 79 (1), 345 (2007).

3. Everaerts, F.M., Beckers, J.L., \& Verheggen, T.P.E.M. Isotachophoresis: Theory, instrumentation, and applications. Elsevier Science \& Technology., (1976).

4. Khurana, T.K. \& Santiago, J.G. Preconcentration, separation, and indirect detection of nonfluorescent analytes using fluorescent mobility markers. Anal. Chem. 80 (1), 279 (2008).

5. Bercovici, M., Kaigala, G.V., Backhouse, C.J., \& Santiago, J.G. Fluorescent carrier ampholytes assay for portable, label-free detection of chemical toxins in tap water. Anal. Chem. 82 (5), 1858 (2010). 
6. Bercovici, M., Kaigala, G.V., \& Santiago, J.G. Method for analyte identification using isotachophoresis and a fluorescent carrier ampholyte assay. Anal. Chem. 82 (5), 2134 (2010).

7. Kaigala, G.V., et al. Miniaturized system for isotachophoresis assays. Lab Chip. 10 (17), 2242 (2010).

8. Chambers, R.D. \& Santiago, J.G. Imaging and quantification of isotachophoresis zones using nonfocusing fluorescent tracers. Anal. Chem. 81 (8), 3022 (2009).

9. Schoch, R.B., Ronaghi, M., \& Santiago, J.G. Rapid and selective extraction, isolation, preconcentration, and quantitation of small RNAs from cell lysate using on-chip isotachophoresis. Lab Chip. 9 (15), 2145 (2009).

10. Persat, A., Marshall, L.A., \& Santiago, J.G. Purification of nucleic acids from whole blood using isotachophoresis. Anal. Chem. 81 (22), 9507 (2009).

11. Bercovici, M., et al. Rapid detection of urinary tract infections using isotachophoresis and molecular beacons. Anal. Chem. 83 (11), 4110 (2011).

12. Marshall, L.A. \& Santiago, J.G. Extraction of DNA from malaria-infected erythrocytes using isotachophoresis. Anal. Chem. 83 (24), 9715 (2011).

13. Persat, A., Chambers, R.D., \& Santiago, J.G. Basic principles of electrolyte chemistry for microfluidic electrokinetics. Part I: Acid-base equilibria and pH buffers. Lab Chip. 9 (17), 2437 (2009).

14. Garcia-Schwarz, G., Bercovici, M., Marshall, L.A., \& Santiago, J.G. Sample dispersion in isotachophoresis. J. Fluid Mech. 1 (1), 1 (2011).

15. Kohlrausch, F. Über concentrations-verschiebungen durch electrolyse im inneren von lösungen und lösungsgemischen. Ann. Phys. 298 (10), 209 (1897).

16. Alberty, R.A. Moving boundary systems formed by weak electrolytes. Theory of simple systems formed by weak acids and bases. J. Am. Chem. Soc. 72 (6), 2361 (1950).

17. Jovin, T.M. Multiphasic zone electrophoresis. I. Steady-state moving-boundary systems formed by different electrolyte combinations. Biochem. 12 (5), 871 (1973).

18. Persat, A. \& Santiago, J.G. MicroRNA profiling by simultaneous selective isotachophoresis and hybridization with molecular beacons. Anal. Chem. (2011). 